\title{
Weapons system portfolio selection based on the contribution rate evaluation of system of systems
}

\author{
DOU Yajie ${ }^{1, *}$, ZHOU Zhexuan ${ }^{1}$, ZHAO Danling ${ }^{1}$, and WEI Yong ${ }^{2}$ \\ 1. College of Systems Engineering, National University of Defense Technology, Changsha 410073, China; \\ 2. Unit 31008 of the PLA, Beijing 100091, China
}

\begin{abstract}
The weapons system portfolio selection problem arises at the equipment demonstration stage and deals with the military application requirements. Further, the contribution rate of the system is one of the important indicators to evaluate the role of a system, which can facilitate the weapons system portfolio selection. Therefore, combining the system contribution rate with system portfolio selection is the focus of this study. It also focuses on calculating the contribution rates of multiple equipment systems with various types of capabilities. The contribution rate is measured by establishing a hierarchical multi-criteria value model from three dimensions. Based on the value model, the feasible portfolios are developed under certain cost constraints and the optimal weapons system portfolios are obtained by using the classification optimization selection strategy. Finally, an illustrative example is presented to verify the feasibility of the proposed model.
\end{abstract}

Keywords: weapon system, system of systems (SoS), portfolio selection, contribution rate evaluation, decision analysis.

DOI: $10.21629 / J S E E .2019 .05 .09$

\section{Introduction}

Markowitz first proposed the modern portfolio theory in 1952, opening a new era of applying mathematical approaches to finance studies [1]. In the field of management science and operations research, portfolio decision theory is a method for resource optimization allocation, which is widely used in project investment [2], product development [3], technology research and development [4], and defense procurement [5]. The project portfolio selection field is another important area where the portfolio selection theory is widely used. Project portfolio selection compares the eligible project portfolio schemes to identify their pros and cons and selects the optimal project portfolio. The methods commonly used include goal planning

Manuscript received September 19, 2018.

*Corresponding author.

This work was supported by the National Key R\&D Program of China (2017YFC1405005) and the National Natural Science Foundation of China (71690233). methods [6], such as 0-1 integer programming [7] and hybrid or dynamic programming. The genetic algorithm, Monte Carlo simulation, and Lagrangian relaxation are often applied to the solution process to determine the feasible projects or super-subsets of capital based on specific resource constraints. From the perspective of a modeling framework, the scenario-based planning method is gradually gaining more attention. It studies foreground probability, describes the uncertainties of the desired future scenario, and develops strategies to match those uncertainties. Christopher James and others introduced scenario analysis and multi-criteria decision theory to project portfolio selection in military and industrial facilities [8].

Portfolio selection is mainly affected by two factors. First, the preferences and attitudes of the decision makers are incorporated in the portfolio selection model considering subjective rationality, irrationality and incomplete rationality, fuzzy stochastic theory, and preference-based decision-making methods. Second, for the investment assets, utility model, stochastic control, and stochastic differential game are comprehensively used to study several problems related to the selection of investment portfolios [9]. The empirical research on project portfolio has developed from two aspects: management that favors project selection and optimization that favors operations [10-12]. It is worth mentioning that studies [13] introduced the synergy and systems theories to the research of aerospace model project portfolio and proposed the strategy-oriented optimization model, which is of high referential significance. More practical applications of project portfolio theory facilitating real life problems can be found in a combinatorial selection model for interruptible projects $[14,15]$, the portfolio selection models considering interactions between projects [16], and environmental and information uncertainty [17].

In the military field, one typical application of portfolio decision making is the wave model for systems evolution 
and capability portfolio model for system of systems (SoS) proposed by the researchers at Purdue University in the United States [18]. However, in the evaluation of weapons system portfolio, most of the studies lack "weapon characteristics", "joint", and "systematic thinking", which are not well distinguished from the general project portfolio selection. Therefore, clarifying the essence of weapons system "portfolio" in the system of weaponry and joint operations and to find a portfolio selection model that fits the characteristics of the weapons system portfolio are still worthy of further study.

In the latest research, weapons and equipment systems are generally evaluated from the perspective of capability, effectiveness, risk, technical feasibility, evolution process, and the impact of new technology introduction on the system [19-22]. The contribution rate of weapons and equipment system is a fairly new concept in the military field. The idea of a contribution rate was first seen in the economic and social fields, such as the contribution rate of science and technology, labor, investment, education, etc. to the economy [23-25] and later developed into other areas of production and life. So far, there have been few applications in the military area and the research mainly focuses on the technology contribution rate [26,27] and equipment contribution rate [28,29].

The research on the contribution rate of weapons system can be roughly categorized into conceptual and methodological analysis. The former investigates the purpose and mode of using equipment contribution rate [30], summarizes the multiple aspects that need to be considered when analyzing the capability of the weapons system and its contribution rate [31], and studies the specific process of assessment of the contribution rate $[32,33]$, etc. The latter focuses on the construction of an evaluation framework quantifying the system contribution rate, which mainly includes the analytic hierarchy process, expert knowledge method, complex network analysis [34], multi-attribute assessment, gray target theory, and the correlation model [30-35].

As mentioned earlier, former studies have failed to combine joint operations and systematic warfare ideas to reflect the contribution rate of the system. They have barely analyzed the contribution rate of weapons and equipment from multi-directional, multi-angle, and multi-level perspectives and there is no unified framework for evaluating the contribution rate. Therefore, it can be concluded that the research on the contribution rate of weapons system is still immature and does not satisfy military needs.

The contribution rate was introduced to the weapons system portfolio selection because it is perfectly suitable to construct the value objective that is the foundation of the optimization in the portfolio selection. The system contribution rate is designed to measure the "function improvement", "performance improvement", "capability improvement", or "effectiveness improvement" that the weapons and equipment system might produce for the entire system. Therefore, the assessment based on the contribution rate of the system can help decision makers weigh the pros and cons of adopting certain systems and develop a weapons system plan with a higher contribution rate.

The rest of this paper is organized as follows. Section 2 defines the contribution rate of a system and describes the problem in this study. Then, the value model based on the contribution rate for the weapons/equipment system portfolio selection is established and the possible solution is given based on multi-objective optimization in Section 3, by which the optimal portfolio can be obtained. Section 4 presents an illustrative case study to examine the performance and feasibility of the proposed model.

\section{Problem description}

\subsection{Preliminaries}

Many studies have examined the weapons/equipment contribution from different viewpoints, but the concept has not been clearly defined and elaborated. In this study, we define that weapons/equipment contribution refers to the capability or operational effectiveness of a weapons/equipment in the system to complete the mission under the given operational conditions. According to this definition, there is a contributory relationship within the system - a directed relationship between contributors and beneficiaries. The contributors are those who make up the overall system and contribute to the performance during the mission. The contributors in this study are the weapons/equipment to be selected. The beneficiaries are those who get benefits from the selected systems, specifically the overall system of systems.

The type of contribution of the weapons/equipment system can be classified as shown in Fig. 1.

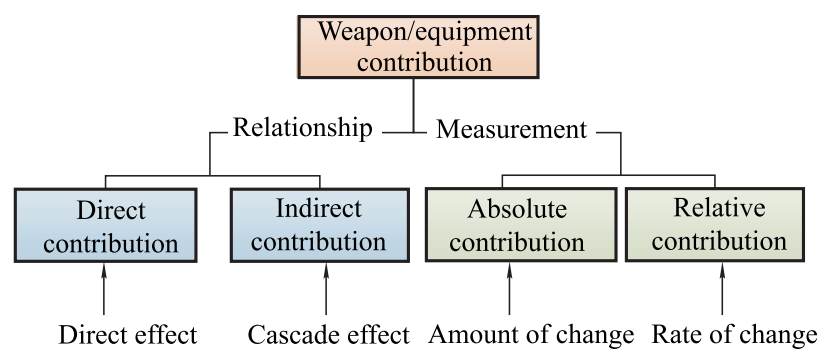

Fig. 1 Weapons/equipment contribution classification

The contribution can be categorized as direct or indirect based on the relationship between the contributors and beneficiaries. The direct contribution of the 
weapons/equipment system refers to the effect directly brought by the weapons/equipment under given operational conditions. For a weapon, the direct contribution can be measured by the proportion of the number of targets damaged to the number of targets. The indirect contribution of the weapons/equipment system refers to the cascade effect on other weapons/equipment and on the overall mission.

The measurement of contribution can be classified into absolute and relative weapons/equipment system contribution. Absolute weapons/equipment system contribution describes the change in the augmented effects by adopting the weapons/equipment in the SoS while the relative weapons/equipment system contribution rate quantifies the change rate.

The definition of weapon/equipment contribution evaluation in this study is given as: the weapons/equipment system contribution evaluation refers to the evaluation of the integrated escalation by introducing multiple new weapons/equipment systems when facing certain missions in future joint combat. The contribution will be judged with respects to "capability improvement", "effectiveness augmentation", or "reserve effect", concerning the attributes of each weapons/equipment system, the internal interaction modes, and the features of the mission tasks.

\subsection{Model framework}

The study aims at a realistic problem of arranging the appropriate weapon development plan for the upcoming operational environment. Even though considerable efforts have been devoted into weapons R\&D, the overall improvement of SoS performance is not outstanding. Further, without proper capability planning, some capabilities overflow and many weapons and equipment projects have been suspended or canceled due to resource and time limitations, resulting in a significant waste of resources and "development failure". This weapons/equipment-oriented development strategy can no longer be provided for today's military needs. Instead, the systemic evaluation of the weapons/equipment contribution to the SoS should be implemented before the production stage to clarify "capability improvement" and "effectiveness augmentation" that each weapons/equipment system would provide in future combat. The contribution of a certain weapon/equipment to the SoS will be assessed respectively from capability, connectivity, and invulnerability.

Capability, the most important attribute of a weapon/ equipment, is the specific reflection of the operational requirements of the mission and a core indicator in the weapon/equipment development. The weapon/equipment capability is mainly determined by the function and tech- nical specification of the weapon/equipment.

In the so-called information war, the weapons/equipment are no longer isolated from each other. The interconnected cooperation between the weapons/equipment systems results in a non-linear growth in combat capability. This connectivity exhibits a dynamic feature, so the directed graph model is applied to study the contribution to the system connectivity, analyze the influence of structure between weapons/equipment on the connectivity, and target the key nodes and factors in the SoS network.

Invulnerability is measured by system redundancy, which means once the essential nodes in the SoS are destroyed, how well can the other weapons/equipment make up for the loss in capabilities and whether they can replace the destroyed system under certain circumstances.

This study constructs a value model from the perspective of the contribution rate of the weapon/equipment to facilitate the weapons/equipment system portfolio selection. As shown in Fig. 2, the proposed model consists of two phases: multi-criteria value model based on the contribution rate and portfolio selection based on multiobjective optimization. In phase 1 , the contribution rate is calculated for capability, connectivity, and invulnerability, respectively, to establish a weapons/equipment portfolio value model. In phase 2 , multi-objective optimization is used to obtain the optimal portfolio.

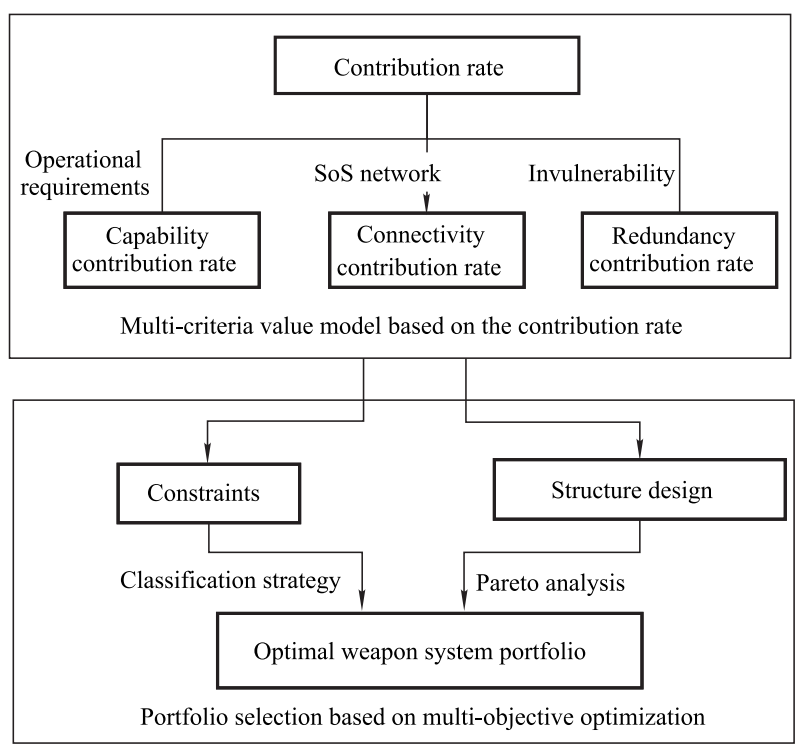

Fig. 2 Weapon portfolio selection framework based on contribution rate evaluation

\section{Portfolio selection based on the contribution rate}

\subsection{Model framework}

This section calculates the contribution rate of each weapon/equipment on three dimensions - capability, con- 
nectivity, and invulnerability. The aggregate result will be the foundation of the establishment of the value function for portfolio selection later.

(i) Capability contribution

The weapons/equipment system capability contribution is calculated by the capability gap method. It is a traditional evaluation method measuring the capability difference of the overall SoS with and without a single equipment. The capability index system of the SoS is established from top to bottom and then the capability of each weapons system is evaluated by standardizing and integrating the index based on the measured values of the weapon/equipment performance as shown in Fig. 3.

The specific assessment framework and process of the capability gap method are as follows: i) establish the structure of the weapons/equipment SoS; ii) determine the capabilities of the weapons system in the assessment and their normalized models; iii) select certain weapons/equipment as the evaluation object; iv) standard- ize the measured values of the capabilities of various types of weapons/equipment and obtain the standardized scores; v) determine the weight of each capability using subjective or objective weighting methods; vi) according to the aggregation relationship between the sub-system and SoS (such as "and", "or", etc.), calculate the evaluation value of the overall capability; vii) compare the results between different selections. This evaluation process is shown in Fig. 4.

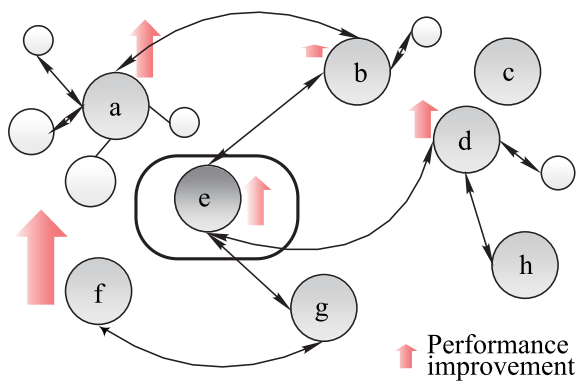

Fig. 3 Schematic diagram of the system capability contribution

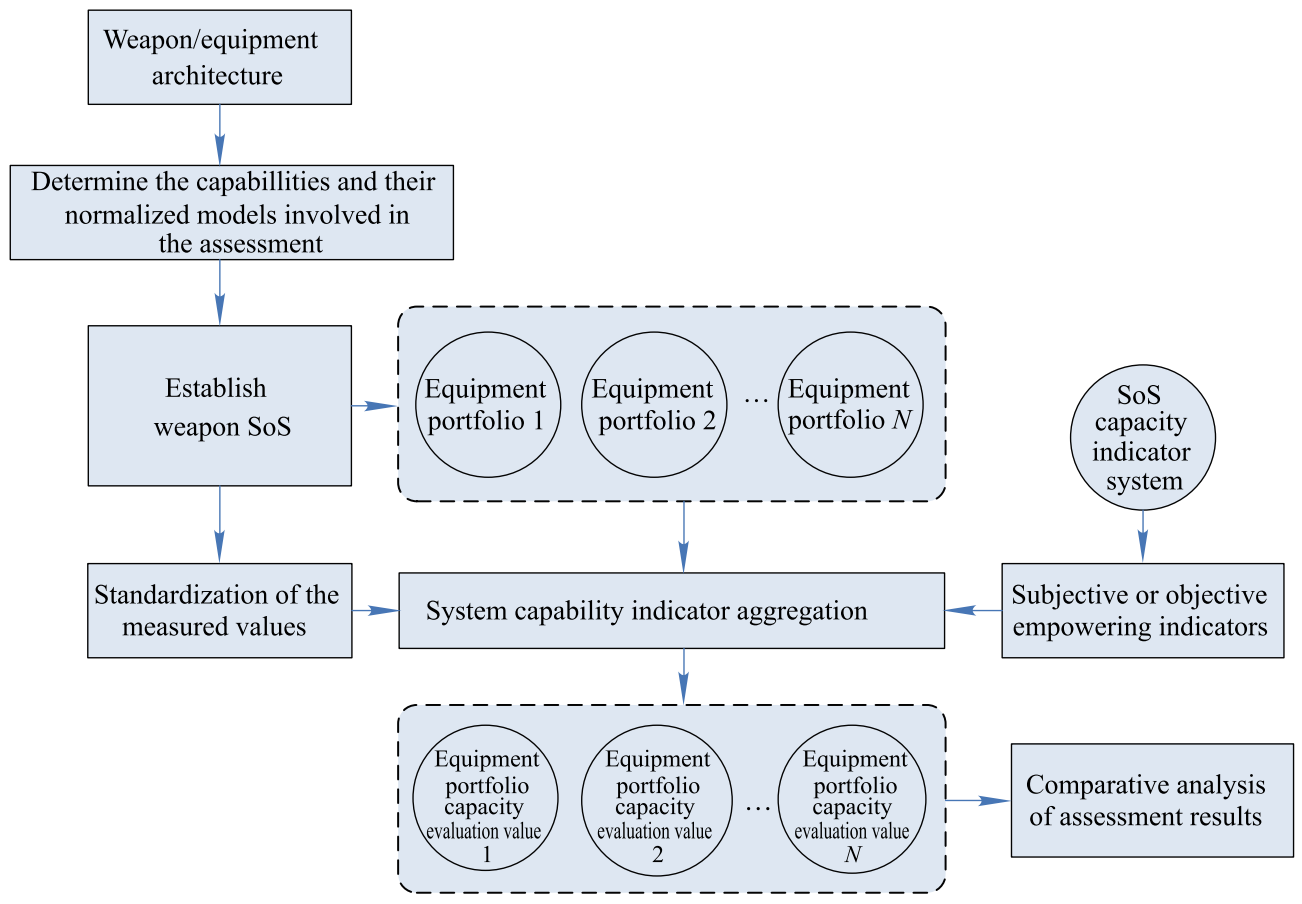

Fig. 4 Capability contribution evaluation process

(ii) Connectivity contribution

The connectivity contribution of the weapon/equipment is evaluated using the connectivity matrix spectral norm and spanning tree-based method, aiming at measuring the overall attributes of the SoS, analyzing the impact of weapons/equipment topology on the connectivity of the SoS and targeting the key nodes and factors in the structural relationship. The illustrative diagram of the con- nectivity contribution of the weapon/equipment system is shown in Fig. 5.

The directed graph model of the weapons/equipment system based on the SoS structure must be established to obtain the corresponding connectivity matrix or the indegree matrix, which is the premise of the connectivity evaluation. The equipment system connectivity contribution assessment framework is shown in Fig. 6. 

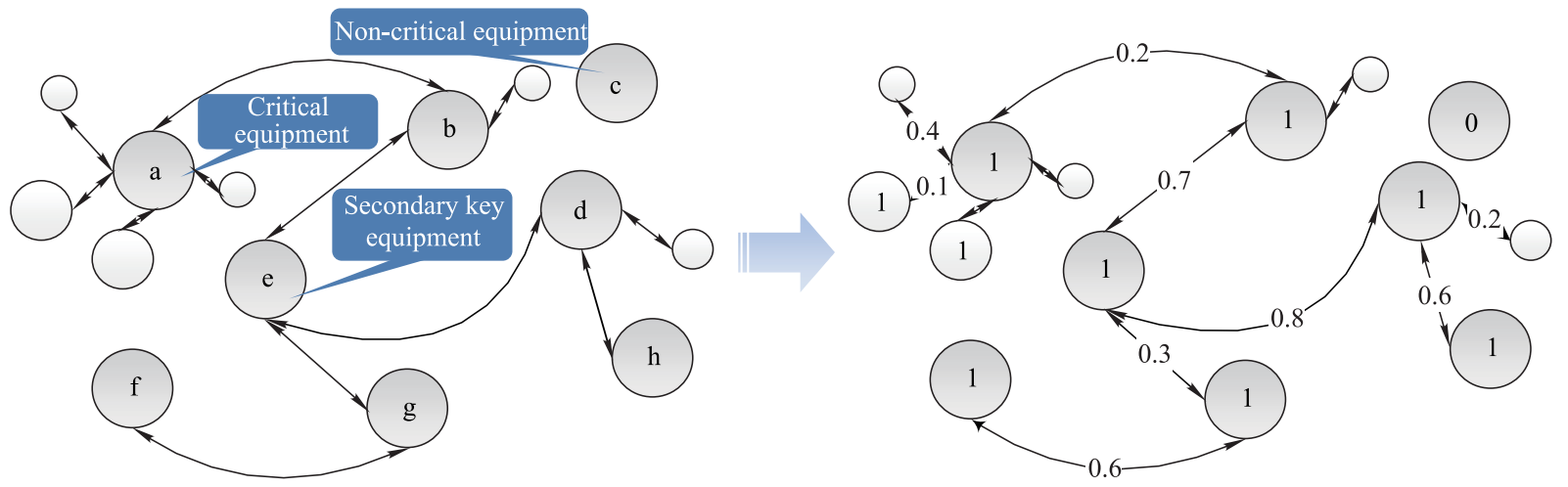

Fig. 5 General framework

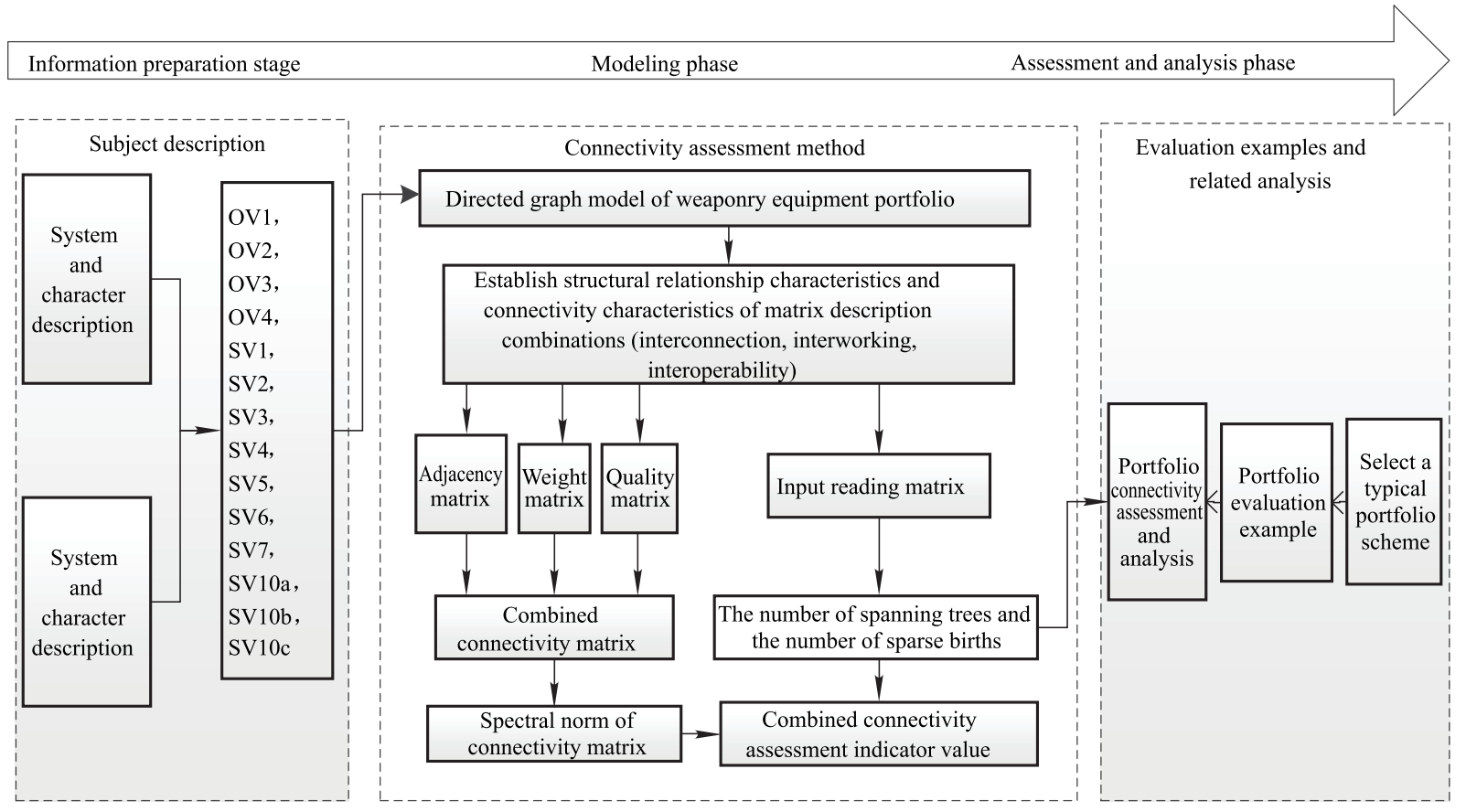

Fig. 6 Equipment system connectivity contribution assessment framework

In the information preparation phase, department of defense architecture framework (DoDAF) is used to acquire the data for the evaluation. System interface view (SV1) and system communication description (SV2) are mainly referred to in terms of connectivity. Specifically, the directed graph model will be constructed according to SV1 and SV2; the interaction quality of the system is determined by SV2.

In the modeling phase, a directed graph model is established with the obtained information to describe the structural relationship features (interconnection, interworking, interoperability characteristics) of the SoS, so that the connectivity matrix and the in-degree matrix can be constructed. These matrices are the numerical representations of the directed graph and by processing the matrix, the characteristics of the matrix can effectively measure the connectivity of the directed graph, that is, evaluate the connectivity of the system. Specifically, the spectral norm of the connectivity matrix can describe the connectivity of the directed graph; the number of the spanning trees of the directed graph can be calculated by the in-degree matrix and with the exhaustive algorithm, all the spanning trees of the directed graph will be generated.

\subsubsection{Construction of the connectivity matrix}

The connectivity matrix $\boldsymbol{C}=f(\boldsymbol{A}, \boldsymbol{W}, \boldsymbol{Q})$ includes the information on the topology of the weapons/equipment SoS, the importance of the interactions between the weapons and equipment, and the interaction quality. $\boldsymbol{A}$ is an adjacency matrix that describes the existence of the interactions between the nodes in the directed graph model of the weapons/equipment $\operatorname{SoS} ; \boldsymbol{W}$ is a weight matrix that 
describes the importance of the interactions between the nodes (the edges in the directed graph) to the connectivity of the SoS; $\boldsymbol{Q}$ is the interaction quality matrix that measures the quality of the interactions between the nodes.

(i) Adjacency matrix $\boldsymbol{A}$

The graph is denoted as $D=(V, E)$, where $V$ is the vertex set and $E$ is the edge set. For $v_{i}, v_{j} \in V$, edge $e_{i j}=\left(v_{i}, v_{j}\right) \in E$ represents an edge pointing from $v_{i}$ to $v_{j}$, which is called the outgoing edge of $v_{i}$ or the incoming edge of $v_{j}$. The number of the outgoing or incoming edges of $v_{i}$ is called out-degree or in-degree, denoted as $d^{+}\left(v_{i}\right)$ or $d^{-}\left(v_{i}\right)$ and $d^{+}\left(v_{i}\right)\left(d^{-}\left(v_{i}\right)\right) \geqslant 0$.

The adjacency matrix of $D$ is denoted as $\boldsymbol{A}(D)=$ $\left(a_{i j}\right)_{n \times n}$, where

$$
a_{i j}=\left\{\begin{array}{ll}
1, & \left(v_{i}, v_{j}\right) \in E \\
0, & \text { Otherwise }
\end{array} .\right.
$$

As seen above, the adjacency matrix $\boldsymbol{A}$ can be directly obtained from the directed graph.

(ii) Weight matrix $\boldsymbol{W}$

Weight matrix $\boldsymbol{W}$ is given as

$$
\begin{gathered}
\sum_{i=1}^{n} \sum_{j=1}^{n} w_{i j}=1 \\
\operatorname{diag}(\boldsymbol{W})=0 .
\end{gathered}
$$

(iii) Interaction quality matrix $Q$

The measurement of the internal information interaction of the SoS is the basis for establishing the interaction quality matrix. It requires evaluating the information exchange quality of intelligence, situation, command, control, etc. between sensor nodes, command and control (C2) nodes, and attack nodes. The related attributes are communication bandwidth, communication reliability, information resolution, transmission speed, and information processing speed. To this end, a standardization and aggregation model is necessary to process this information.

\subsubsection{Connectivity evaluation based on matrix spectral norm}

After the connectivity matrix is established, the evaluation of the connectivity can be converted into the operation on the connectivity matrix. The norm indicates the distance from any point in the Euclidean space to the origin of the coordinate, which can be used to measure the "size" of the point. If we consider the $n$th order matrix $\boldsymbol{C}$ as one point in the Euclidean space $\mathbf{R}^{m \times n}$, the matrix norm $\|\boldsymbol{C}\|$ can be used as the measurement of the matrix $C$. The commonly used matrix norm is spectral norm, given as

$$
\left\|\boldsymbol{C}^{*}\right\|_{2}=\max \left\{\lambda \mid \lambda \text { is eigenvalue of } \boldsymbol{C}^{* \mathrm{~T}} \cdot \boldsymbol{C}^{*}\right\}=
$$

$$
\sqrt{\lambda_{\max }\left(\boldsymbol{C}^{* \mathrm{~T}} \cdot \boldsymbol{C}^{*}\right)}=\sigma_{1} .
$$

Spectral norm $\left\|C^{*}\right\|_{2}$ is calculated according to the eigenvalues of the matrix, which is a more comprehensive measurement of the matrix information and better characterizes the matrix.

The spectral norm of the connectivity matrix clearly satisfies the non-negative feature of system connectivity and follows monotonicity. Further, when merging two unrelated matrices, the spectral norm of the new matrix remains less than the original two. The proofs on the above features are given as follows.

Proposition 1 Assume $\boldsymbol{A}=\left[a_{i j}\right]_{n \times n}$ and $\boldsymbol{B}=$ $\left[b_{i j}\right]_{m \times m}$ are two unrelated system connectivity matrices. The connectivity matrix of the new system composed of the two is

$$
\boldsymbol{C}=\left[\begin{array}{cc}
\boldsymbol{A} & 0 \\
0 & \boldsymbol{B}
\end{array}\right]_{m+n},
$$

then

$$
\left\|\boldsymbol{C}^{*}\right\|_{2}=\max \left\{\|\boldsymbol{A}\|_{2},\|\boldsymbol{B}\|_{2}\right\} .
$$

Proof Let $\lambda_{1} \geqslant \lambda_{2} \geqslant \cdots \geqslant \lambda_{n} \geqslant 0$ be the eigenvalues of $\boldsymbol{A}^{\mathrm{T}} \cdot \boldsymbol{A}$. Since $\boldsymbol{A}^{\mathrm{T}} \cdot \boldsymbol{A}$ is a Hermite matrix, there is an $n$-order unitary matrix, which makes

$$
\boldsymbol{U}_{\boldsymbol{A}}^{\mathrm{H}} \boldsymbol{A}^{\mathrm{H}} \boldsymbol{A} \boldsymbol{U}_{\boldsymbol{A}}=\operatorname{diag}\left(\lambda_{1}, \lambda_{2}, \ldots, \lambda_{n}\right) .
$$

Let $\mu_{1} \geqslant \mu_{2} \geqslant \cdots \geqslant \mu_{n} \geqslant 0$ be the eigenvalues of $\boldsymbol{B}^{\mathrm{T}} \cdot \boldsymbol{B}$, then here is an $n$-order unitary matrix $\boldsymbol{U}_{\boldsymbol{B}}$, which makes

$$
\boldsymbol{U}_{\boldsymbol{B}}^{\mathrm{H}} \boldsymbol{B}^{\mathrm{H}} \boldsymbol{B} \boldsymbol{U}_{\boldsymbol{B}}=\operatorname{diag}\left(\mu_{1}, \mu_{2}, \ldots, \mu_{n}\right) .
$$

Then,

$$
\begin{gathered}
{\left[\begin{array}{cc}
\boldsymbol{U}_{\boldsymbol{A}}^{\mathrm{H}} & 0 \\
0 & \boldsymbol{U}_{\boldsymbol{B}}^{\mathrm{H}}
\end{array}\right] \boldsymbol{C}^{\mathrm{T}} \boldsymbol{C}\left[\begin{array}{cc}
\boldsymbol{U}_{\boldsymbol{A}} & 0 \\
0 & \boldsymbol{U}_{\boldsymbol{B}}
\end{array}\right]=} \\
{\left[\begin{array}{cc}
\boldsymbol{U}_{\boldsymbol{A}}^{\mathrm{H}} & 0 \\
0 & \boldsymbol{U}_{\boldsymbol{B}}^{\mathrm{H}}
\end{array}\right]\left[\begin{array}{cc}
\boldsymbol{A} & 0 \\
0 & \boldsymbol{B}
\end{array}\right]^{\mathrm{T}}\left[\begin{array}{cc}
\boldsymbol{A} & 0 \\
0 & \boldsymbol{B}
\end{array}\right]\left[\begin{array}{cc}
\boldsymbol{U}_{\boldsymbol{A}} & 0 \\
0 & \boldsymbol{U}_{\boldsymbol{B}}
\end{array}\right]=} \\
{\left[\begin{array}{cc}
\boldsymbol{U}_{\boldsymbol{A}}^{\mathrm{H}} & 0 \\
0 & \boldsymbol{U}_{\boldsymbol{B}}^{\mathrm{H}}
\end{array}\right]\left[\begin{array}{cc}
\boldsymbol{A}^{\mathrm{T}} & 0 \\
0 & \boldsymbol{B}^{\mathrm{T}}
\end{array}\right]\left[\begin{array}{cc}
\boldsymbol{A} & 0 \\
0 & \boldsymbol{B}
\end{array}\right]\left[\begin{array}{cc}
\boldsymbol{U}_{\boldsymbol{A}} & 0 \\
0 & \boldsymbol{U}_{\boldsymbol{B}}
\end{array}\right]=} \\
{\left[\begin{array}{cc}
\boldsymbol{U}_{\boldsymbol{A}}^{\mathrm{H}} \boldsymbol{A}^{\mathrm{T}} \boldsymbol{A} \boldsymbol{U}_{\boldsymbol{A}} & 0 \\
0 & \boldsymbol{U}_{\boldsymbol{B}}^{\mathrm{H}} \boldsymbol{B}^{\mathrm{T}} \boldsymbol{B} \boldsymbol{U}_{\boldsymbol{B}}
\end{array}\right] .}
\end{gathered}
$$

Obviously, $\left[\begin{array}{cc}\boldsymbol{U}_{\boldsymbol{A}} & 0 \\ 0 & \boldsymbol{U}_{\boldsymbol{B}}\end{array}\right]$ is also a unitary matrix and its transposed matrix is $\left[\begin{array}{cc}\boldsymbol{U}_{\boldsymbol{A}} & 0 \\ 0 & \boldsymbol{U}_{\boldsymbol{B}}\end{array}\right]^{\mathrm{H}}=\left[\begin{array}{cc}\boldsymbol{U}_{\boldsymbol{A}}^{\mathrm{H}} & 0 \\ 0 & \boldsymbol{U}_{\boldsymbol{B}}^{\mathrm{H}}\end{array}\right]$.

Then,

$$
\begin{aligned}
\boldsymbol{U}_{\boldsymbol{A}}^{\mathrm{H}} \boldsymbol{A}^{\mathrm{H}} \boldsymbol{A} \boldsymbol{U}_{\boldsymbol{A}} & =\operatorname{diag}\left(\lambda_{1}, \lambda_{2}, \ldots, \lambda_{n}\right) \\
\boldsymbol{U}_{\boldsymbol{B}}^{\mathrm{H}} \boldsymbol{B}^{\mathrm{H}} \boldsymbol{B} \boldsymbol{U}_{\boldsymbol{B}} & =\operatorname{diag}\left(\mu_{1}, \mu_{2}, \ldots, \mu_{n}\right) .
\end{aligned}
$$


Therefore, $\left[\begin{array}{cc}\boldsymbol{U}_{\boldsymbol{A}}^{\mathrm{H}} & 0 \\ 0 & \boldsymbol{U}_{\boldsymbol{B}}^{\mathrm{H}}\end{array}\right] \boldsymbol{C}^{\mathrm{T}} \boldsymbol{C}\left[\begin{array}{cc}\boldsymbol{U}_{\boldsymbol{A}} & 0 \\ 0 & \boldsymbol{U}_{\boldsymbol{B}}\end{array}\right]$ is also a diagonal matrix of $\operatorname{diag}\left(\lambda_{1}, \lambda_{2}, \ldots, \lambda_{n}, \mu_{1}, \mu_{2}, \ldots, \mu_{n}\right)$.

According to the properties of the spectral norm,

$$
\begin{gathered}
\left\|\boldsymbol{C}^{*}\right\|_{2}=\max \left\{\lambda_{1}, \lambda_{2}, \ldots, \lambda_{n}, \mu_{1}, \mu_{2}, \ldots, \mu_{n}\right\}= \\
\max \left\{\lambda_{1}, \mu_{1}\right\}=\max \left\{\|\boldsymbol{A}\|_{2},\|\boldsymbol{B}\|_{2}\right\} .
\end{gathered}
$$

Lemma 1 Suppose $\boldsymbol{A}$ is an $n$-order positive matrix. Its eigenvalues are $\lambda_{1} \leqslant \lambda_{2} \leqslant \cdots \leqslant \lambda_{n}$, then, for any $n$th order vector $\boldsymbol{X}$, the following relationship exists:

$$
\boldsymbol{X}^{\prime} \lambda_{1} \boldsymbol{X} \leqslant \boldsymbol{X}^{\prime} \boldsymbol{A} \boldsymbol{X} \leqslant \boldsymbol{X}^{\prime} \lambda_{n} \boldsymbol{X}
$$

Proof Assume that $\boldsymbol{P}_{1}, \boldsymbol{P}_{2}, \ldots, \boldsymbol{P}_{n}$ are the corresponding feature vectors of the eigenvalue $\lambda_{i}(i=1,2, \ldots, n)$. Since $\boldsymbol{P}_{1}, \boldsymbol{P}_{2}, \ldots, \boldsymbol{P}_{n}$ are the $n$ orthogonal basis vectors in the $n$-dimensional Euclidean space, there are $n$ real numbers $\alpha_{i}(i=1,2, \ldots, n), \boldsymbol{X}=\sum_{i=1}^{n} \alpha_{i} \boldsymbol{P}_{i}$.

$$
\begin{aligned}
& \boldsymbol{X}^{\prime} \lambda_{1} \boldsymbol{X}=\left(\sum_{i=1}^{n} \alpha_{i} \boldsymbol{P}_{i}^{\prime}\right) \boldsymbol{A}\left(\sum_{i=1}^{n} \alpha_{i} \boldsymbol{P}_{i}\right)= \\
& \sum_{i=1}^{n} \alpha_{i}^{2} \boldsymbol{P}_{i}^{\prime} \boldsymbol{A} \boldsymbol{P}_{i}+\sum_{i \neq j}\left(2 \alpha_{i} \alpha_{j} \boldsymbol{P}_{i}^{\prime} \boldsymbol{A} \boldsymbol{P}_{j}\right) .
\end{aligned}
$$

Note that $\boldsymbol{A P}_{i}=\lambda_{i} \boldsymbol{P}_{i}$ and $\boldsymbol{P}_{i}(i=1,2, \ldots, n)$ is the base vector. Then, the right side of (11) can be rewritten as

$$
\begin{gathered}
\boldsymbol{X}^{\prime} \boldsymbol{A} \boldsymbol{X}=\sum_{i=1}^{n} \lambda_{i} \alpha_{i}^{2} \boldsymbol{P}_{i}^{\prime} \boldsymbol{P}_{i}+\sum_{i \neq j}\left(2 \lambda_{i} \alpha_{i} \alpha_{j} \boldsymbol{P}_{j} \boldsymbol{P}_{i}^{\prime}\right)= \\
\sum_{i=1}^{n} \lambda_{i} \alpha_{i}^{2} \boldsymbol{P}_{i} \boldsymbol{P}_{i}^{\prime} .
\end{gathered}
$$

Because $\lambda_{1} \leqslant \lambda_{2} \leqslant \cdots \leqslant \lambda_{n}$, so $\sum_{i=1}^{n} \lambda_{1} \alpha_{i}^{2} \boldsymbol{P}_{i} \boldsymbol{P}_{i}^{\prime} \leqslant$ $\sum_{i=1}^{n} \lambda_{i} \alpha_{i}^{2} \boldsymbol{P}_{i} \boldsymbol{P}_{i}^{\prime} \leqslant \sum_{i=1}^{n} \lambda_{n} \alpha_{i}^{2} \boldsymbol{P}_{i} \boldsymbol{P}_{i}^{\prime}$, which can be written as $\boldsymbol{X}^{\prime} \lambda_{1} \boldsymbol{X} \leqslant \boldsymbol{X}^{\prime} \boldsymbol{A} \boldsymbol{X} \leqslant \boldsymbol{X}^{\prime} \lambda_{n} \boldsymbol{X}$.

Proposition 2 Assume $\boldsymbol{A}=\left[a_{i j}\right]_{n \times n}$ is a connectivity matrix, $\|\boldsymbol{A}\|_{2}$ monotonically increases as $a_{i j}(i \neq j)$ monotonically increases.

Proof Assume one non-diagonal element of the connectivity matrix $\boldsymbol{A}=\left[a_{i j}\right]_{n \times n}$ is $a_{p q}$ and let $a_{p q}^{\prime}=$ $a_{p q}+\Delta_{p q}, \Delta_{i j}>0$. Construct a matrix $\boldsymbol{A}^{\prime}=\left(a_{i j}^{\prime}\right)_{n \times n}$ where $a_{i j}^{\prime}=a_{i j}(i \neq p$ and $j \neq q), a_{p q}^{\prime}=a_{p q}+\Delta_{p q}$.

First, the induction method is used to prove $\Delta=$ $\boldsymbol{A}^{\prime \mathrm{T}} \boldsymbol{A}^{\prime}-\boldsymbol{A}^{\mathrm{T}} \boldsymbol{A}$ is semi-positive.

(i) When $n=2, \boldsymbol{A}=\left[\begin{array}{cc}0 & a_{12} \\ a_{21} & 0\end{array}\right]$ and $\boldsymbol{A}^{\prime}=$ $\left[\begin{array}{cc}0 & a_{12}+\Delta_{12} \\ a_{21} & 0\end{array}\right]$ or $\boldsymbol{A}^{\prime}=\left[\begin{array}{cc}0 & a_{12} \\ a_{21}+\Delta_{21} & 0\end{array}\right]$.

$$
\text { If } \begin{aligned}
\boldsymbol{A}^{\prime} & =\left[\begin{array}{cc}
0 & a_{12}+\Delta_{12} \\
a_{21} & 0
\end{array}\right], \text { it can be calculated that } \\
\Delta & =\boldsymbol{A}^{\prime \mathrm{T}} \boldsymbol{A}^{\prime}-\boldsymbol{A}^{\mathrm{T}} \boldsymbol{A}=\left[\begin{array}{cc}
0 & 0 \\
0 & 2 a_{12} \Delta_{12}+\Delta_{12}^{2}
\end{array}\right] .
\end{aligned}
$$

Clearly, it is semi-positive.

It is the same with $\boldsymbol{A}^{\prime}=\left[\begin{array}{cc}0 & a_{12} \\ a_{21}+\Delta_{21} & 0\end{array}\right]$. Thus when $n=2, \boldsymbol{A}^{\prime \mathrm{T}} \boldsymbol{A}^{\prime}-\boldsymbol{A}^{\mathrm{T}} \boldsymbol{A}$ is semi-positive.

(ii) Assume that $\boldsymbol{A}^{\prime \mathrm{T}} \boldsymbol{A}^{\prime}-\boldsymbol{A}^{\mathrm{T}} \boldsymbol{A}$ are semi-positive when $n<m(m>2)$.

When $n=m+1$, it is easy to prove that the $k$-order principal minors of $\boldsymbol{A}^{\prime \mathrm{T}} \boldsymbol{A}^{\prime}-\boldsymbol{A}^{\mathrm{T}} \boldsymbol{A}$ are semi-positive if $k \leqslant m$. For $k=m+1, \operatorname{det}\left(\boldsymbol{A}^{\prime \mathrm{T}} \boldsymbol{A}^{\prime}-\boldsymbol{A}^{\mathrm{T}} \boldsymbol{A}\right)=0$. Thus, $\boldsymbol{A}^{\prime \mathrm{T}} \boldsymbol{A}^{\prime}-\boldsymbol{A}^{\mathrm{T}} \boldsymbol{A}$ is also semi-positive when $n=m+1$.

From (1) and (2), $\forall n \geqslant 2, \boldsymbol{A}^{\prime \mathrm{T}} \boldsymbol{A}^{\prime}-\boldsymbol{A}^{\mathrm{T}} \boldsymbol{A}$ is semipositive.

Let $\lambda_{\max }$ be the biggest eigenvalue of $\boldsymbol{A}^{\mathrm{T}} \boldsymbol{A}, \boldsymbol{X}_{\max }$ is the eigenvector corresponding to $\lambda_{\max }$ of $\boldsymbol{A} \cdot \lambda_{\max }^{\prime}$ is the biggest eigenvalue of $\boldsymbol{A}^{\prime \mathrm{T}} \boldsymbol{A}^{\prime}$ and $\Delta=\boldsymbol{A}^{\prime \mathrm{T}} \boldsymbol{A}^{\prime}-\boldsymbol{A}^{\mathrm{T}} \boldsymbol{A}$.

From Lemma 1,

$$
\begin{gathered}
\boldsymbol{X}_{\max }^{\prime} \lambda_{\max } \boldsymbol{X}_{\max }=\boldsymbol{X}_{\max }^{\prime}\left(\boldsymbol{A}^{\mathrm{T}} \boldsymbol{A}\right) \boldsymbol{X}_{\max }= \\
\boldsymbol{X}_{\max }^{\prime}\left(\boldsymbol{A}^{\prime \mathrm{T}} \boldsymbol{A}^{\prime}-\Delta\right) \boldsymbol{X}_{\max }= \\
\boldsymbol{X}_{\max }^{\prime} \boldsymbol{A}^{\prime \mathrm{T}} \boldsymbol{A}^{\prime} \boldsymbol{X}_{\max }-\boldsymbol{X}_{\max }^{\prime} \Delta \boldsymbol{X}_{\max }
\end{gathered}
$$

Then,

$$
\begin{gathered}
\boldsymbol{X}_{\max }^{\prime} \lambda_{\max }^{\prime} \boldsymbol{X}_{\max } \geqslant \boldsymbol{X}_{\max }^{\prime}\left(\boldsymbol{A}^{\prime \mathrm{T}} \boldsymbol{A}^{\prime}\right) \boldsymbol{X}_{\max }= \\
\boldsymbol{X}_{\max }^{\prime} \lambda_{\max }^{\prime} \boldsymbol{X}_{\max }-\boldsymbol{X}_{\max }^{\prime} \Delta \boldsymbol{X}_{\max } \geqslant \boldsymbol{X}_{\max }^{\prime} \lambda_{\max }^{\prime} \boldsymbol{X}_{\max } .
\end{gathered}
$$

Hence, $\lambda_{\max }^{\prime} \geqslant \lambda_{\max }$.

In summary, the above characteristics show that the spectral norm of the connectivity matrix is suitable to serve as an indicator to evaluate the connectivity of the weapons/equipment $\mathrm{SoS}$.

\subsubsection{Redundancy contribution}

Consider a situation where $N$ different types of weapons/equipment cooperate to generate a single capability as illustrated in Fig. 7. The redundancy of a certain weapon/equipment is $100 \% / N$ if it can continue to offer the capability when the key node is destroyed or fails, else $0 \%$.

In the situation where there is no cooperation and all the $N$ weapons/equipment can individually generate the same capability, the redundancy of a certain weapon/equipment is $100 \%$ if it can continue to offer the capability when the key node is destroyed or fails, else $0 \%$.

In this study, the case where the weapon/equipment contributes redundancy to multiple capabilities has been simplified. Only one-on-one redundancy contribution is considered and there are no effects between different capabilities. 


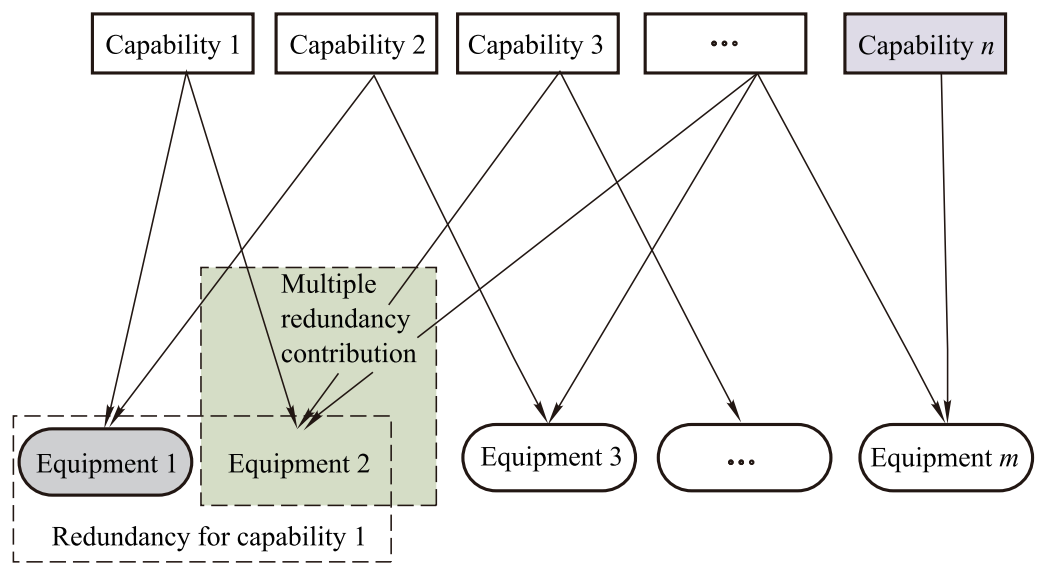

Fig. 7 Schematic diagram of the redundancy contribution

\subsection{Value model based on the contribution rates}

An ideal system portfolio is a group of weapons/equipment that have the best capability, connectivity, and redundancy contribution. Therefore, the system portfolio selection is a multi-objective optimization balancing the three contributions of the portfolio candidates.

In the optimization, the objective value is constructed with the aggregation of the contribution rates. The aggregating logic of the three rates depends on realistic scenarios and specific needs, which can be divided into three types:

(i) Average value

$$
\widetilde{C}=C_{\text {cap }}+C_{\text {con }}+C_{\text {red }}
$$

where $\widetilde{C}$ is the comprehensive contribution rate, $C_{\text {cap }}$, $C_{\text {con }}, C_{\text {red }}$ are the capability, connectivity, and redundancy contribution rate respectively.

(ii) Weighted average

$$
\widetilde{C}=w_{1} C_{\text {cap }}+w_{2} C_{\text {con }}+w_{3} C_{\text {red }}
$$

where $w_{1}, w_{2}, w_{3}$ are the weights of the capability, connectivity, and redundancy contribution rate, respectively, which are usually provided by experts.

(iii) Max value

$$
\widetilde{C}=\max \left\{C_{\text {cap }}, C_{\text {con }}, C_{\text {red }}\right\}
$$

In reality, cost and resources are limited in the system demonstration and production process. Moreover, to guarantee combat performance, a sufficient amount of capabilities is required. Therefore, portfolio selection is a constrained optimization and the constraints involve the cost budget and capability requirements.

In summary, the value model based on the system contribution rate consists of two parts: the value model and the constraints.

\subsection{Feasible portfolio}

To generate the optimization solutions, multi-objective integer programming is used. Firstly, the feasible portfolio set is achieved with the given constraints. Secondly, the main structure to identify the optimal weapons/equipment system portfolios is defined. Finally, the optimal portfolio is generated by multi-objective integer programming.

Generally, portfolio $P_{S}$ is the sub-set of candidates set $S^{*}$. All the feasible portfolios can be expressed as

$$
P^{*}=\left\{P_{S} \mid P_{S} \subseteq S^{*}, s \in \mathbf{N}^{+}\right\} .
$$

Feasible portfolio set $P_{F} \subseteq P^{*}$ is subject to different constraints, such as resource availability, budget, and capability requirements. These constraints are usually modeled by a set of linear inequalities, as follows:

$$
P_{F}^{*}=\left\{P_{S} \in P^{*} \mid \boldsymbol{C}\left(P_{S}\right) \leqslant \boldsymbol{B}, \underset{P_{s}}{N(\boldsymbol{C})}=v, s \in \mathbf{N}^{+}\right\}
$$

where the matrix $\boldsymbol{C} \in \mathbf{R}^{q \times N_{P_{s}}(s)}$ and $\boldsymbol{B} \subseteq \mathbf{R}^{q},{ }_{P_{S}}^{N(\boldsymbol{C})}$ are used to describe the number of the capabilities provided by the portfolio $P_{S}$.

\subsection{Optimal portfolio}

Among the feasible portfolios, the one that can maximize the value, which is the comprehensive contribution rate, will be identified as the optimal portfolio. However, the computational and time complexities of finding all the optimal portfolios are too high. Previous studies have provided some relatively efficient ways to find the entire optimal portfolio set from a feasible portfolio space. Owing to the characteristics of multi-objectives, one commonly used method is to find all the Pareto optimal solutions with multi-objective integer programming.

In general, optimization is implemented through pairwise comparisons between system portfolios. However, 
when there are many portfolios, the difficulty of conducting pairwise comparison will grow exponentially and become time consuming. The cost-based classification strategy proposed by Kung [36] and Deb [37] have proven to be more efficient in identifying Pareto optimal solutions from many feasible portfolios. The algorithm to generate the optimal portfolio set based on the classification strategy is presented as follows.

Step 1 Suppose the number of feasible portfolios in $P_{F}^{*}$ is $M, M<p \in \mathbf{N}^{+}$. The enumeration of feasible portfolios in the descending order of cost is $P_{F}^{*}=$ $\left\{P_{1}, P_{2}, \ldots, P_{M}\right\}$. If $s<s^{\prime}$, then $C\left(P_{s}\right) \geqslant C\left(P_{s^{\prime}}\right)$.

Step 2 Initialize the optimal portfolio set $s, P_{T R}^{* 0} \leftarrow \phi$ and $s \leftarrow 0$.

Step 3 Implement the iteration with all $P_{s}<P_{F}^{*}$ in the ascending order of $s$. When $s<M$,

(i) the subscript $s$ is increased to $s+1$;

(ii) if $\exists P_{s^{\prime}} \in P_{N D}^{*_{s}-1}$ satisfies $P_{s^{\prime}} \succ P_{s}$, then $P_{T R}^{* s} \leftarrow$ $P_{T R}^{* s-1}$ and go to Step 3;

(iii) $P_{T R}^{* s} \leftarrow\left\{P_{s}\right\} \cup\left\{P_{s^{\prime}} \in P_{T R}^{* s-1} \mid P_{s} \nsucc P_{s^{\prime}}\right\}$ and go to Step 3.

Step $4 P_{T R}^{*} \leftarrow P_{T R}^{* M}, P_{T R}^{*}$ is the final optimal portfolio set.

This algorithm reduces the number of pairwise comparisons by sorting the feasible portfolios in the descending order of their cost. It is worth noting that the algorithm used in this paper is an improvement of the method proposed by Liesiö, Mild, and Salo [38]. The feasible portfolio set is treated as the initial solution space rather than all the feasible portfolios and the classification strategy is conducted flexibly more than once, which makes the algorithm more efficient by reducing the number of comparisons.

\section{Illustration example}

\subsection{Example description}

Taking the Air Police weapon system and the corresponding equipment portfolio as an example, eight system candidates are represented by $\mathrm{S} 1-\mathrm{S} 8$. The calculation of the system's capability contribution, connectivity contribution, and redundancy contribution, and the selection of weapons/equipment system portfolio based on the contribution rate will be illustrated in detail. The original SoS consists of six weapons/equipment and their capabilities levels as shown in Table 1.

At this time, the capability requirements in the operational scenario are listed in Table 2.

The cost budgets of the eight weapons/equipment candidates to be developed and their essential capabilities are shown in Table 3. The capability categories include reconnaissance capability, decision-making capability, com- mand capability, and strike capability.

Table 1 Capability types and levels of the original equipment system

\begin{tabular}{ccc}
\hline Equipment & Capability type & $\begin{array}{c}\text { Capability } \\
\text { level (1-9) }\end{array}$ \\
\hline E1 & Reconnaissance ability & 7 \\
\hline \multirow{2}{*}{ E2 } & Reconnaissance ability & 6 \\
& Decision-making capacity & 5 \\
\hline E3 & Decision-making capacity & 7 \\
\hline \multirow{2}{*}{ E4 } & Decision-making capacity & 6 \\
& Command ability & 6 \\
\hline E5 & Command ability & 8 \\
\hline E6 & Strike ability & 7 \\
\hline
\end{tabular}

Table 2 Levels of operational requirements for each capability

\begin{tabular}{cc}
\hline Capability type & Operational demand level \\
\hline Reconnaissance ability & 9 \\
Decision-making capacity & 7 \\
Command ability & 7 \\
Strike ability & 8 \\
\hline
\end{tabular}

Table 3 Cost, capability type, and capability level of the candidates

\begin{tabular}{|c|c|c|c|}
\hline Equipment & Cost & Capability type & $\begin{array}{r}\text { Capability } \\
\text { level }(1-9)\end{array}$ \\
\hline S1 & 1200 & Reconnaissance ability & 8 \\
\hline S2 & 1700 & Decision-making capacity & 8 \\
\hline \multirow{2}{*}{ S3 } & \multirow{2}{*}{3600} & Reconnaissance ability & 8 \\
\hline & & Decision-making capacity & 8 \\
\hline \multirow{3}{*}{ S4 } & \multirow{3}{*}{4000} & Reconnaissance ability & 6 \\
\hline & & Decision-making capacity & 8 \\
\hline & & Command ability & 7 \\
\hline \multirow{2}{*}{ S5 } & \multirow{2}{*}{2500} & Decision-making capacity & 7 \\
\hline & & Command ability & 7 \\
\hline S6 & 1800 & Command ability & 9 \\
\hline S7 & 3000 & Strike ability & 8 \\
\hline S8 & 3500 & Strike ability & 9 \\
\hline
\end{tabular}

\subsection{Example calculation and results analysis}

\subsubsection{Capacity contribution calculation}

The maximum capability level in the existing SoS is chosen as the value of that capability of the SoS, which is $V^{\mathrm{i}}=\max \left\{V_{1}^{\mathrm{i}}, V_{2}^{\mathrm{i}}, \ldots, V_{n}^{\mathrm{i}}\right\}$. Then, the reconnaissance capability value that the SoS is equipped with is $V^{1}=$ $\max \{7,6\}=7$. Similarly, the decision-making, command, and strike capability values are 7,8 and 7 , respectively.

The comprehensive capability assessment of the current SoS is obtained by the weighted aggregation of the ratios of the four capabilities to the capability requirements, which becomes the basic value. The bigger the ratio, the higher the level of satisfaction of the capability requirements and the higher the assessment. 


$$
\begin{gathered}
V=\sum_{j=1}^{4} w_{j} V_{j}=0.25 \times \frac{7}{9}+ \\
0.25 \times \frac{7}{7}+0.25 \times \frac{8}{7}+0.25 \times \frac{7}{8}=0.949
\end{gathered}
$$

The changes in the capability of the SoS by introducing candidates S1 and S8 into the system and the capability contribution rate of this portfolio will be explained in detail. S1 provides level 8 reconnaissance capability. Therefore, by introducing $\mathrm{S} 1$, the reconnaissance capability value of the $\operatorname{SoS}$ becomes $V^{1}=\max \{7,6,8\}=$ 8. The strike capability of S8 is 9; after adopting S8, the strike capability of the system becomes $V^{4}=$ $\max \{7,9\}=9$.

The comprehensive capability assessment of the SoS when $\mathrm{S} 1$ and $\mathrm{S} 8$ are added to the system is

$$
\begin{aligned}
V= & \sum_{j=1}^{4} w_{j} V_{j}=0.25 \times \frac{8}{9}+0.25 \times \frac{7}{7}+ \\
& 0.25 \times \frac{8}{7}+0.25 \times \frac{9}{8}=1.039 .
\end{aligned}
$$

By adding the candidates $\mathrm{S} 1$ and $\mathrm{S} 8$, the comprehensive capability increases from 0.949 to 1.039 , which means that the capability contribution rate of the portfolio $\{\mathrm{S} 1, \mathrm{~S} 8\}$ is

$$
C_{\text {cap }}=\frac{1.039-0.949}{0.949} \times 100 \%=9.48 \% \text {. }
$$

According to the above calculation, the capability contribution rates of each candidate are shown in Table 4.

Table 4 Capability contribution rate of candidates

\begin{tabular}{ccc}
\hline $\begin{array}{c}\text { Preparing to } \\
\text { develop } \\
\text { equipment }\end{array}$ & $\begin{array}{c}\text { Capacity } \\
\text { contribution } \\
\text { rate/\% }\end{array}$ & $\begin{array}{c}\text { Specific } \\
\text { improvement ability }\end{array}$ \\
\hline S1 & 2.92 & Reconnaissance ability \\
S2 & 3.77 & Decision-making capacity \\
S3 & 6.69 & Reconnaissance and decision making \\
S4 & 3.77 & Decision-making capacity \\
S5 & 0 & None \\
S6 & 3.77 & Command ability \\
S7 & 3.29 & Strike ability \\
S8 & 6.59 & Strike ability \\
\hline
\end{tabular}

Among them, though S5 has multiple capabilities, none of them is outstanding, hence, its capability contribution rate is $0 \%$. However, that does not necessarily mean that S5 is worthless. S5 exhibits advantages and importance in the calculations of the connectivity and redundancy contribution rates.

\subsubsection{Connectivity contribution}

Assume that the connected relationship between the weapons and equipment shown in Fig. 8 exists in the original SoS. The direction and interaction quality between them can be seen from the figure. For example, the interaction quality between the weapons systems E6 and E4 is 0.9 and the direction is $\mathrm{E} 4 \rightarrow \mathrm{E} 6$.

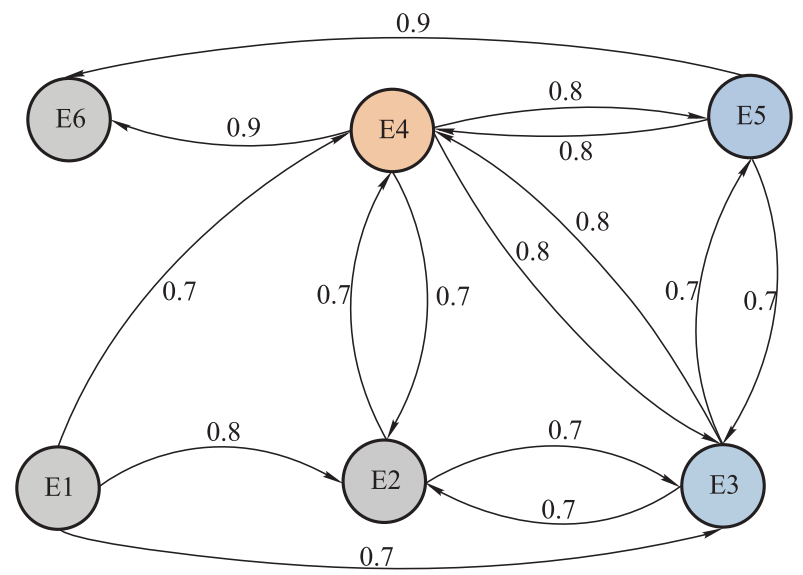

Fig. 8 Connected relationship between weapons and equipment of the original equipment system

The information in Fig. 9 can be expressed by a matrix based on the directed graph and adjacency matrix. The adjacency matrix $\boldsymbol{A}_{1}$ of the original SoS is

$$
\boldsymbol{A}_{1}=\left[\begin{array}{llllll}
0 & 1 & 1 & 1 & 0 & 0 \\
0 & 0 & 1 & 1 & 0 & 0 \\
0 & 1 & 0 & 1 & 1 & 0 \\
0 & 1 & 1 & 0 & 1 & 1 \\
0 & 0 & 1 & 1 & 0 & 1 \\
0 & 0 & 0 & 0 & 0 & 0
\end{array}\right]
$$

where 1 denotes connected and 0 denotes unconnected.

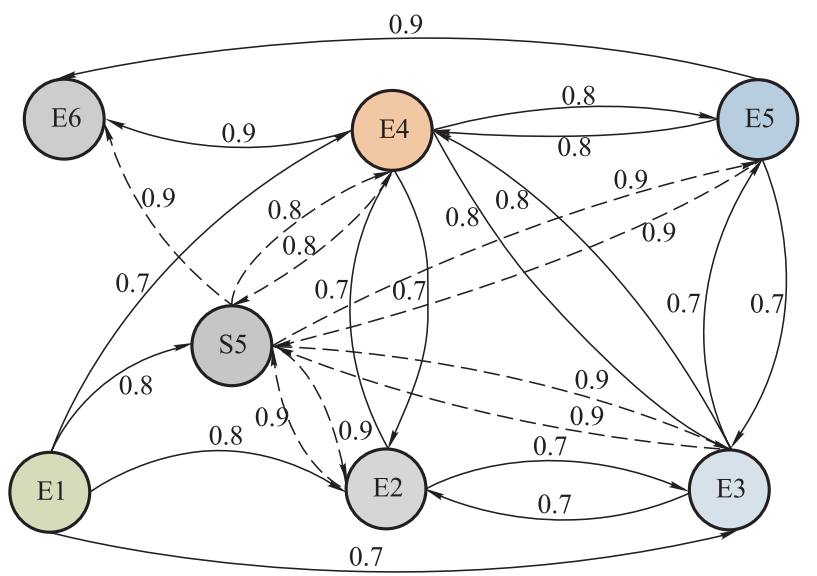

Fig. 9 Connected relationship after adopting S5

The weight matrix of the system after standardization is 
given by experts.

$\overline{\boldsymbol{W}}_{1}=\left[\begin{array}{ccccccc}0 & 0.01 & 0.02 & 0.02 & 0.03 & 0.03 & 0.03 \\ 0.01 & 0 & 0.03 & 0.03 & 0.02 & 0.02 & 0.03 \\ 0.02 & 0.03 & 0 & 0.03 & 0.02 & 0.03 & 0.03 \\ 0.03 & 0.03 & 0.03 & 0 & 0.03 & 0.03 & 0.03 \\ 0.03 & 0.02 & 0.02 & 0.03 & 0 & 0.02 & 0.02 \\ 0.03 & 0.02 & 0.02 & 0.03 & 0.02 & 0 & 0.03 \\ 0.03 & 0.03 & 0.03 & 0.03 & 0.02 & 0.03 & 0\end{array}\right]$

The interaction quality matrix $Q_{1}$ is

$$
\boldsymbol{Q}_{1}=\left[\begin{array}{ccccccc}
0 & 0.8 & 0.7 & 0.7 & 0 & 0 & 0 \\
0 & 0 & 0.7 & 0.7 & 0.9 & 0 & 0 \\
0 & 0.7 & 0 & 0.8 & 0.7 & 0 & 0 \\
0 & 0.7 & 0.8 & 0 & 0.8 & 0.9 & 0 \\
0 & 0.9 & 0.7 & 0.8 & 0 & 0.9 & 0 \\
0 & 0 & 0 & 0 & 0 & 0 & 0 \\
0 & 0 & 0 & 0 & 0 & 0 & 0
\end{array}\right]
$$

The connectivity matrix $\boldsymbol{C}_{1}$ is

$$
\begin{gathered}
\boldsymbol{C}_{1}=f\left(\boldsymbol{A}_{1}, \overline{\boldsymbol{W}}_{1}, \boldsymbol{Q}_{1}\right)= \\
{\left[\begin{array}{cccccc}
0 & 0.0086 & 0.0126 & 0.0126 & 0 & 0 \\
0 & 0 & 0.0227 & 0.0176 & 0 & 0 \\
0 & 0.0227 & 0 & 0.0201 & 0.0126 & 0 \\
0 & 0.0176 & 0.0201 & 0 & 0.0201 & 0.0291 \\
0 & 0 & 0.0126 & 0.0201 & 0 & 0.0162 \\
0 & 0 & 0 & 0 & 0 & 0
\end{array}\right] .}
\end{gathered}
$$

Clearly, the spectral norm of matrix $C_{1}$ is 0.0568 .
Now consider adding S5 to the SoS. The new connectivity relationship is shown in Fig. 9.

Similarly,

$$
\boldsymbol{A}_{2}=\left[\begin{array}{lllllll}
0 & 1 & 1 & 1 & 0 & 0 & 1 \\
0 & 0 & 1 & 1 & 0 & 0 & 1 \\
0 & 1 & 0 & 1 & 1 & 0 & 1 \\
0 & 1 & 1 & 0 & 1 & 1 & 1 \\
0 & 0 & 1 & 1 & 0 & 1 & 1 \\
0 & 0 & 0 & 0 & 0 & 0 & 0 \\
0 & 1 & 1 & 1 & 1 & 1 & 0
\end{array}\right]
$$$$
\overline{\boldsymbol{W}}_{2}=\left[\begin{array}{ccccccc}
0 & 0.01 & 0.02 & 0.02 & 0.03 & 0.03 & 0.03 \\
0.01 & 0 & 0.03 & 0.03 & 0.02 & 0.02 & 0.03 \\
0.02 & 0.03 & 0 & 0.03 & 0.02 & 0.03 & 0.03 \\
0.02 & 0.03 & 0.03 & 0 & 0.03 & 0.03 & 0.03 \\
0.03 & 0.02 & 0.02 & 0.03 & 0 & 0.02 & 0.02 \\
0.03 & 0.02 & 0.03 & 0.03 & 0.02 & 0 & 0.03 \\
0.03 & 0.03 & 0.03 & 0.03 & 0.02 & 0.03 & 0
\end{array}\right] .
$$

The interaction quality matrix of the new SoS is

$$
\boldsymbol{Q}_{2}=\left[\begin{array}{ccccccc}
0 & 0.8 & 0.7 & 0.7 & 0 & 0 & 0.9 \\
0 & 0 & 0.7 & 0.7 & 0.9 & 0 & 0.9 \\
0 & 0.7 & 0 & 0.8 & 0.7 & 0 & 0.9 \\
0 & 0.7 & 0.8 & 0 & 0.8 & 0.9 & 0.9 \\
0 & 0.9 & 0.7 & 0.8 & 0 & 0.9 & 0.9 \\
0 & 0 & 0 & 0 & 0 & 0 & 0.9 \\
0 & 0.9 & 0.9 & 0.9 & 0.9 & 0.9 & 0
\end{array}\right] .
$$

The connectivity matrix is

$$
\boldsymbol{C}_{2}=f\left(\boldsymbol{A}_{2}, \overline{\boldsymbol{W}}_{2}, \boldsymbol{Q}_{2}\right)=\left[\begin{array}{ccccccc}
0 & 0.0086 & 0.0126 & 0.0126 & 0 & 0 & 0.0227 \\
0 & 0 & 0.0227 & 0.0176 & 0 & 0 & 0.0291 \\
0 & 0.0227 & 0 & 0.0201 & 0.0126 & 0 & 0.0227 \\
0 & 0.0176 & 0.0201 & 0 & 0.0201 & 0.0291 & 0.0259 \\
0 & 0 & 0.0126 & 0.0201 & 0 & 0.0126 & 0.0126 \\
0 & 0 & 0 & 0 & 0 & 0 & 0 \\
0 & 0.0291 & 0.0227 & 0.0291 & 0.0162 & 0.0227 & 0
\end{array}\right]
$$

Then, the spectral norm of the new connectivity matrix is 0.0883 .

The linear spectral normalization function is used to normalize the obtained spectral values (the maximum is 0.1 and the minimum is 0 ) and the connectivity levels of the two SoS are 56.8 and 88.3. The adoption of S5 raises the connectivity level of the SoS by $55.46 \%$.

Similarly, all the connectivity contribution rates of the candidates can be calculated. The connected relationship between the candidates and the existing SoS after adoption are shown in Fig. 10.

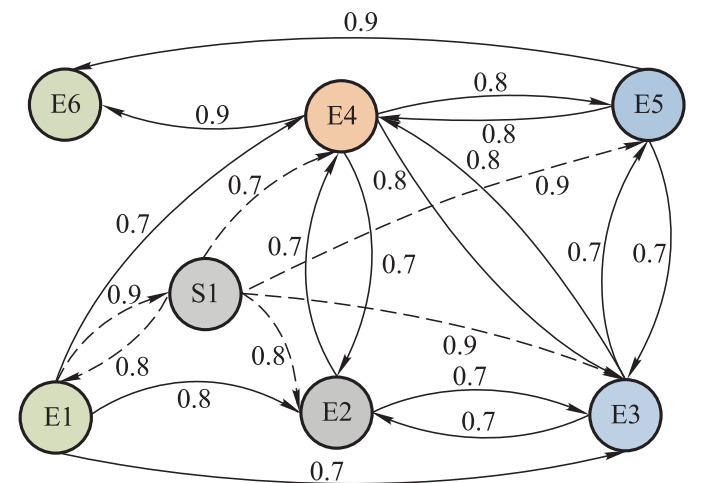

(a) Commected relationship after adopting $\mathrm{S} 1$ 


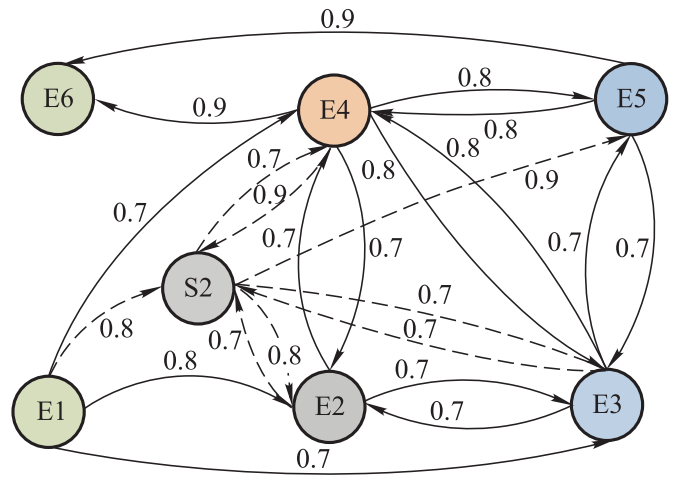

(b) Commected relationship after adopting S2

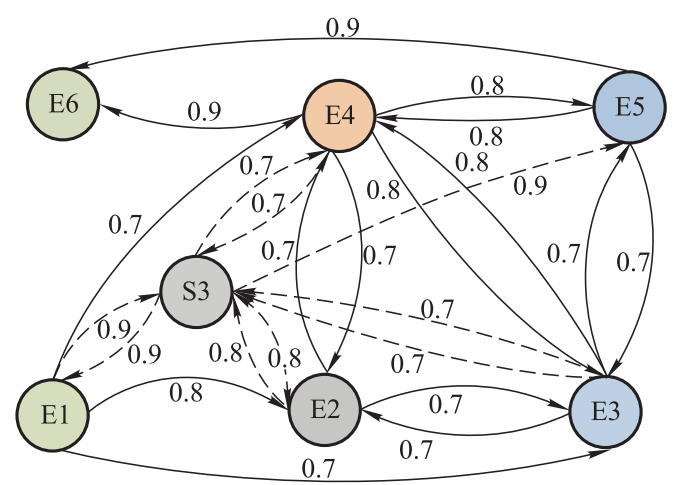

(c) Commected relationship after adopting S3

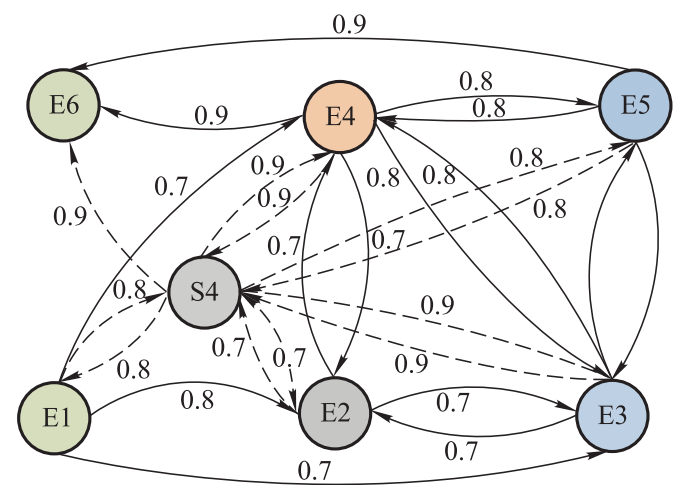

(d) Commected relationship after adopting S4

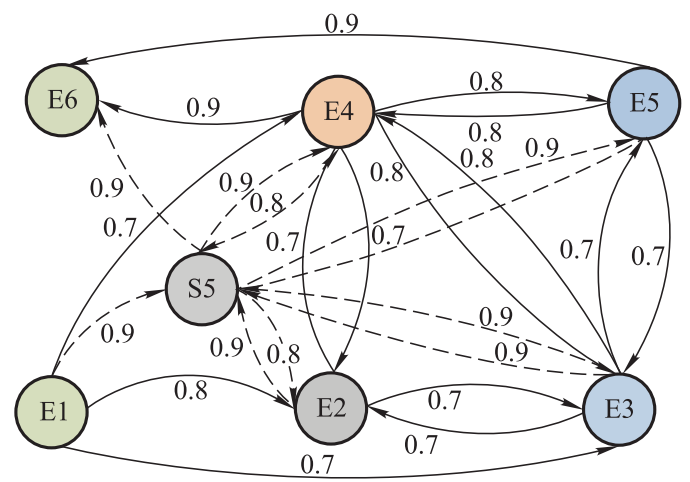

(e) Commected relationship after adopting S5

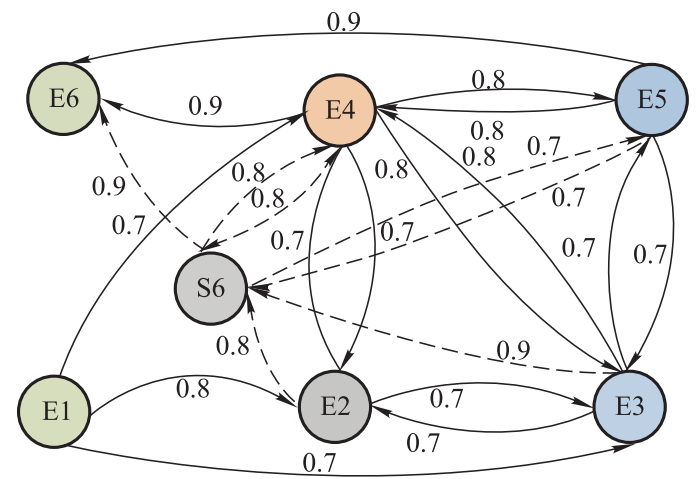

(f) Commected relationship after adopting S6

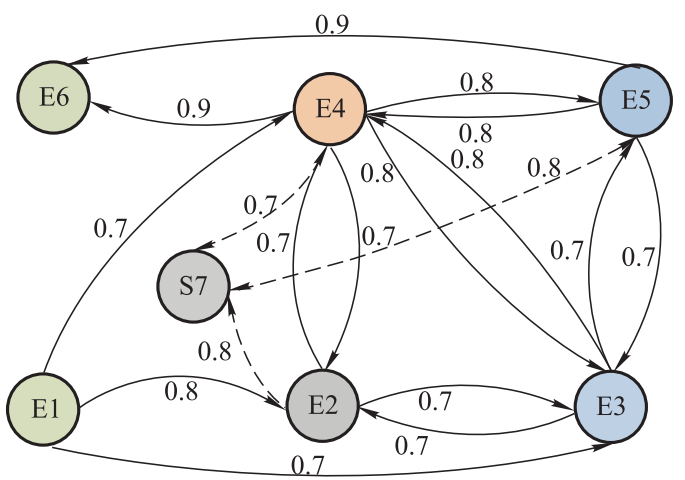

(g) Commected relationship after adopting S7

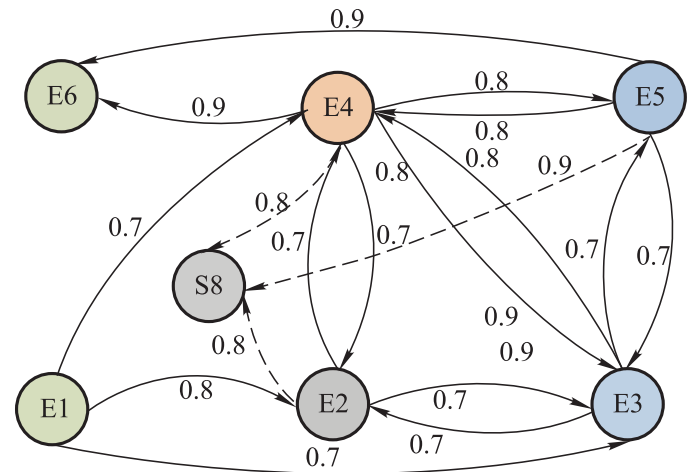

(h) Commected relationship after adopting $\mathrm{S} 8$

Fig. 10 Connected relationship between the candidates and the existing SoS after adoption of S1 - S8

The results of the connectivity contribution rates are shown in Table 5.

Table 5 Connectivity contribution rates of the candidates

\begin{tabular}{cc}
\hline Candidate & Connectivity contribution rate/\% \\
\hline S1 & 23.98 \\
S2 & 36.80 \\
S3 & 36.72 \\
S4 & 52.22 \\
S5 & 55.49 \\
S6 & 31.39 \\
S7 & 7.43 \\
S8 & 11.71 \\
\hline
\end{tabular}


Since S4 and S5 have multiple capabilities, they can connect with more weapons/equipment in the SoS network. Therefore, there is no doubt that they have a high connectivity contribution rate. The candidates S7 and S8 are both strike equipment that can only receive information from the command node, so their connectivity contribution rates are the least.

\subsubsection{Redundancy contribution}

Suppose $\mathrm{S} 4$ is selected and the supporting relationships between each weapon/equipment and the corresponding capability is shown in Fig. 11.

The "reconnaissance capability" can be supported by E1, E2, and S4; the "decision capability" can be supported by E2, E3, E4, and S4; the "command capability" can be supported by E4, E5, and S4; and the "strike capability" is only supported by E6. According to the definition of redundancy contribution in Section 3.1, the redundancy contribution of S4 to reconnaissance capability, decision-making capability, command capability, and strike capability are $33.33 \%, 25 \%, 33.33 \%$, and $0 \%$ respectively. The comprehensive redundancy contribution rate of S4 is the sum of all four, $91.66 \%$. Similarly, the redundancy contribution rate of each candidate can be calculated. The results are shown in Table 6.

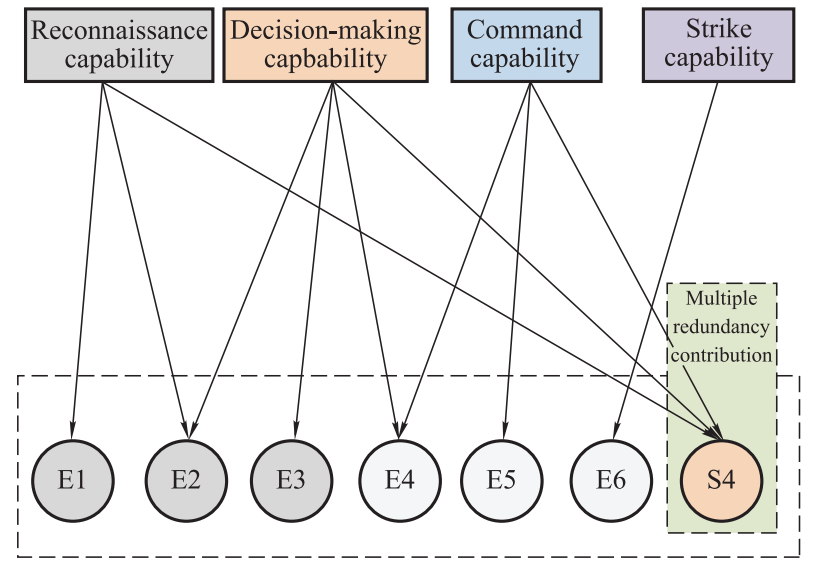

Fig. 11 Supporting relationships between the weapons/equipment and their capabilities

Table 6 Redundancy contribution rate of each candidate to be developed

\begin{tabular}{cc}
\hline Candidate to be developed & Redundancy contribution rate/\% \\
\hline S1 & 33.33 \\
S2 & 25 \\
S3 & 58.33 \\
S4 & 91.66 \\
S5 & 58.33 \\
S6 & 33.33 \\
S7 & 50 \\
S8 & 50 \\
\hline
\end{tabular}

Since S4 can support multiple capabilities reconnaissance, decision-making, and command-it has the highest redundancy contribution rate. This means that the invulnerability of the SoS is more likely maximized by developing S4. On the contrary, since S2 can only provide decision-making capability and there are already three equipment in the original system supporting the same, it has the lowest redundancy contribution rate.

The three dimensions of the contribution measurement of each candidate obtained through the above calculation and the cost to develop each candidate are listed in Table 7. With the proposed portfolio selection algorithm in Section 3.4, the final portfolio selection results are shown in Table 8.

Table 7 Contribution rates of each candidate and cost to develop

\begin{tabular}{ccccc}
\hline $\begin{array}{c}\text { Planned } \\
\text { development } \\
\text { equipment }\end{array}$ & $\begin{array}{c}\text { Capacity } \\
\text { contribution } \\
\text { rate/\% }\end{array}$ & $\begin{array}{c}\text { Connected } \\
\text { contribution } \\
\text { rate/\% }\end{array}$ & $\begin{array}{c}\text { Redundancy } \\
\text { contribution } \\
\text { rate/\% }\end{array}$ & Cost \\
\hline S1 & 2.92 & 23.98 & 33.33 & 1200 \\
S2 & 3.77 & 36.80 & 25 & 1700 \\
S3 & 6.69 & 36.72 & 58.33 & 3600 \\
S4 & 3.77 & 52.22 & 91.66 & 4000 \\
S5 & 0 & 55.49 & 58.33 & 2500 \\
S6 & 3.77 & 31.39 & 33.33 & 1800 \\
S7 & 3.29 & 7.43 & 50 & 3000 \\
S8 & 6.59 & 11.71 & 50 & 3500 \\
\hline
\end{tabular}

Table 8 List of 30 non-inferior weapon system portfolios

\begin{tabular}{cc||cc||cc}
\hline Portfolio & System & Portfolio & System & Portfolio & System \\
\cline { 3 - 5 } P1 & S7,S8 & P11 & S2,S8 & P21 & S1,S4,S6 \\
P2 & S6,S8 & P12 & S2,S7 & P22 & S1,S3,S4 \\
P3 & S4,S7 & P13 & S2,S6 & P23 & S2,S3 \\
P4 & S4,S6 & P14 & S2,S5 & P24 & S2,S3,S6 \\
P5 & S4,S5 & P15 & S1,S8 & P25 & S4,S8 \\
P6 & S3,S8 & P16 & S1,S7,S8 & P26 & S3,S4 \\
P7 & S5 & P17 & S1,S6,S8 & P27 & S1,S2,S4 \\
P8 & S5,S8 & P18 & S1,S5,S8 & P28 & S1,S2,S3 \\
P9 & S5,S7 & P19 & S1,S2,S7 & P29 & S1,S5,S7 \\
P10 & S2,S4 & P20 & S1,S2,S6 & P30 & S1,S4,S7 \\
\hline
\end{tabular}

\subsection{Results analysis}

(i) Influence of the contribution rates on the portfolio selection results

It can be seen from the results that the contribution rates vary significantly with candidates. Taking S5 and S7 as examples, the contribution rates of the former are $0 \%$, $55.49 \%$, and $58.33 \%$, respectively, while $3.29 \%, 7.43 \%$, and $50 \%$, respectively, for the latter. Further, the improvement on the capability of SoS is not outstanding with a maximum of $6.69 \%$ by S3, and a minimum of $0 \%$ by S5.

Nevertheless, thanks to the rich interconnection and interoperability between the weapons/equipment, the $\mathrm{SoS}$ connectivity exhibits remarkable improvement. Especially 
for S5, its connectivity contribution rate is as high as $55.49 \%$.

From this analysis, the candidates with better performance on the three contribution rates appear more frequently in the non-inferior solutions and affect the final portfolio selection results.

(ii) The impact of cost constraints

Although there are only eight candidates, the number of feasible solutions reach up to 255 theoretically. However, the number of the final non-inferior solutions is 30 and the maximal number of candidates in one portfolio is only 3 . This means that the cost constraint plays an important role in reducing the solution space in the portfolio selection.

As seen from the results, the candidates with higher cost, such as S3 and S4, appear less frequently in the noninferior solutions than the ones with lower cost, such as S1 and $\mathrm{S} 2$.

In this study, the overall cost has been simplified as the accumulation of the cost of each candidate. In fact, the calculation of the overall cost might be more complicated in reality, rather than simply summing up the resource sharing. Besides, the cost constraints can be regarded as more than just monetary constraints, which means all the consumptions and resource costs associated with the weapons/equipment should be transferred into the constraints.

\section{Conclusions}

This study benefits weapons/equipment portfolio selection by introducing the concept of contribution rate to the value model. Three different dimensions of the system contribution rate - the capability, connectivity, and redundancy contribution rates - are proposed to construct a multiobjective value function. In the optimization, the feasible solution space is reduced by the cost constraints and the optimal portfolios are selected based on the Pareto optimal analysis with the modified cost-based classification strategy. To verify the feasibility of the model, an illustrative example is used to analyze the characteristics of the acquired portfolios.

This paper mainly focuses on the evaluation of the contribution rate of one side of an SoS. However, a real war is a confrontation with the opponent's SoS. Therefore, SoS confrontation should be further studied, where the dynamic confrontation processes among multi-SoS are embedded into the assessment process.

\section{References}

[1] MARKOWITZ H. Portfolio selection. Journal of Finance, 1952, 7(1): 71-91.

[2] A C X, LI Z F, WANG F. Optimal investment strategy under time-inconsistent preferences and high-water mark contract. Operations Research Letters, 2016, 44(2): 212-218.

[3] MULLER S, HAASE K. On the product portfolio planning problem with customer-engineering interaction. Operations Research Letters, 2016, 44(3): 390-393.

[4] NATH P, RAMAKRISHAN R. Environmental management practices, environmental technology portfolio, and environmental commitment: a content analytic approach for UK manufacturing firms. International Journal of Production Economics, 2015, 171(1/3): 427-437.

[5] KANGASPUNTA J, LIESIO J, SALO A. Cost-efficiency analysis of weapon system portfolios. European Journal of Operational Research, 2012, 223(1): 264-275.

[6] LEE J, KANG S H, ROSENBERGER J, et al. A hybrid approach of goal programming for weapon systems selection. Computers \& Industrial Engineering, 2010, 58(3): 521 - 527.

[7] ZHOU D Y, HUANG H L, TENG C Y, et al. Project selection of robust portfolio models with incomplete information. Journal of Finance and Investment Analysis, 2012, 1(2): 157-199.

[8] CHRISTOPHER W, KARVETSKI Y, JAMES H, et al. Scenario and multiple criteria decision analysis for energy and environmental security of military and industrial installations. Integrated Environmental Assessment and Management, 2010, 7(2): $228-236$.

[9] WU H. Dynamic portfolio selection theory and its application in time-varying financial markets. Changsha: Hunan University, 2016. (in Chinese)

[10] AN H G. Research on the theoretical framework and method of project portfolio selection. Xi' an: Northwestern Polytechnical University, 2007. (in Chinese)

[11] TANG Y F, BAI L Z, GUO Y T. Research on project portfolio selection based on strategy orientation. Project Management Technology, 2012, 10(2): 21-25.

[12] YANG Y, YANG S L, HU X J. Selection of new product development project portfolio based on strategic consistency. Systems Engineering - Theory and Practice, 2014, 34(4): 964-970. (in Chinese)

[13] WANG X B. Strategy-oriented optimization and collaborative research of aerospace model project portfolio. Xi'an: Northwestern Polytechnical University, 2016. (in Chinese)

[14] LI X M, WEI H J, YAN J X, et al. Research on the model of interruptible project portfolio selection under resource constraints. China Management Science, 2016, 24(11): 40-46. (in Chinese)

[15] LI X M, LIU Z L, ZHAO Q H. The local sensitivity analysis of project portfolio selection problem with divisibility. Systems Engineering — Theory and Practice, 2016, 36(7): 18161825. (in Chinese)

[16] TAO S, SHENG Z W, ZHU J B. robust decision making of project selection with interaction uncertain project interactions. Chinese Journal of Management Science, 2017, 25(4): $190-196$.

[17] LI X M, ZHONG Z M, ZHAO Q H, et al. Robust optimization for project portfolio selection problem with divisibility under information uncertainty. Systems Engineering - Theory and Practice, 2017, 37(11): 2908 - 2917. (in Chinese)

[18] DAVENDRALINGAM N, MANE M, DELAURENTIS D. Capability and development risk management in systemof-systems architectures: a portfolio approach to decisionmaking. Proc. of the 9th Annual Acquisition Research Symposium, 2012: $25-36$.

[19] GOROD A, SAUSER B, BOARDMAN J. System-of-systems engineering management: a review of modern history and a path forward. IEEE Systems Journal, 2008, 2(4): 484-499.

[20] LI S K, MOGI G, HUI K S. A fuzzy analytic hierarchy pro- 
cess (AHP)/data envelopment analysis (DEA) hybrid model for efficiently allocating energy $R \& D$ resources: in the case of energy technologies against high oil prices. Renewable \& Sustainable Energy Reviews, 2013, 21: $347-355$.

[21] CHENG B, JIANG J, TAN Y J, et al. A novel approach for WSoS capability requirement satisfactory degree evaluation using evidential reasoning. Systems Engineering - Theory and Practice, 2011, 31(11): 2210-2216. (in Chinese)

[22] GUAN Q B, YU X H. Research on evaluation of equipment's contribution to system war fighting. Journal of Equipment Academy, 2015, 26(3): 1-5.

[23] ENGLISH L, POPP J, MILLER W. Economic contribution of the agricultural sector to the Arkansas economy in 2012. Research report (Arkansas Agricultural Experiment Station), 2014.

[24] HUNT A, STONE I. Assessing contribution of the north east of England's higher education sector to the regional economy. Regional \& Urban Modeling, 2014, 3(2): 1334-1339.

[25] CAMPBELL H F. Measuring the contribution of education to labor productivity in a developing economy. International Journal of Education Economics \& Development, 2009, 1(2): 156.

[26] CHANG L L, CHENG B, LI M, et al. Multi-view and network modeling for technology system of systems. Proc. of the IEEE International Conference on System Science and Engineering, 2012: $297-301$.

[27] CHANG L L, LU Y, ZHAO Q S, et al. Technology system of systems: concepts and hierarchical structure. Proc. of the IEEE International Conference on System of Systems Engineering, 2012: $269-274$.

[28] LI J C, YANG K W, FU C X, et al. An operational efficiency evaluation method for weapon system-of-systems combat networks based on operation loop. Proc. of the IEEE International Conference on System of Systems Engineering, 2014: 219223.

[29] LI J C. Research on networked modeling and operational effectiveness evaluation methods of weapon system-of-systems. Proc. of the International Conference on Management and Engineering, 2014: 45-69. (in Chinese)

[30] LEUNG C. Capability-based planning for Australia's national security. Security Challenges, 2010, 6(3): 78-96.

[31] YU Q Z, ZANG Y C, ZHANG J, et al. Research on operational effectiveness evaluation method of equipment system based on overall effect. System Simulation Technology, 2011, 7(3): $183-189$

[32] SHUY, TAN Y J, LIAO L C. Evaluation of combat capability of weapon equipment system based on capability requirements. Ordnance Automation, 2009, 28(11): 17-19.

[33] GE H Z, ZHENG A H. Evaluation of technical contribution based on improved algorithm of grey target theory. Scientific Decision, 2012(2): $27-36$.

[34] LI J C, YANG K Z, ZHANG X K, et al. Equipment contribution degree evaluation method based on combat network of weapon system-of-systems. Complex Systems and Complexity Science, 2016, 13(3): 1-7.
[35] LI J C. Research on the contribution of equipment system based on combat network model. Changsha, China: National University of Defense Technology, 2015.

[36] KUNG H, LUCCIO F, PREPARATA F. On finding the maxima of a set of vectors. Journal of the Association for Computing Machinery, 1975, 22(4): 469-476.

[37] DEB K. Multi-objective optimization using evolutionary algorithms. Chichester: John Wiley \& Sons, 2001.

[38] IESIO J, MOLD P, SALO A. Preference programming for robust portfolio modeling and project selection. European Journal of Operational Research, 2007, 181(3): 1488-1505.

\section{Biographies}

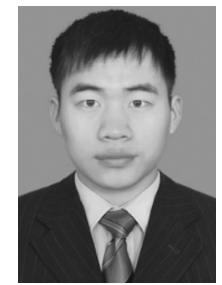

DOU Yajie was born in 1987. He received his $\mathrm{Ph} . \mathrm{D}$. degree in management science and engineering from the National University of Defense Technology (NUDT), Changsha, China. In 2014-2015, he was a visiting researcher in the KU Leuven in Belgium. He teaches decision-making analysis and system-of-systems engineering in the College of Systems Engineering at NUDT. His main research interests are system portfolio selection, decision making, and effectiveness evaluation.

E-mail: yajiedou_nudt@163.com

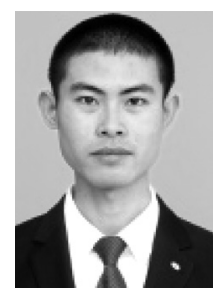

ZHOU Zhexuan was born in 1994. He is a graduate student of National University of Defense Technology University. His research interests are systems engineering, portfolio selection analysis, group decision-making, and fuzzy decision-making.

E-mail: 372985928@qq.com

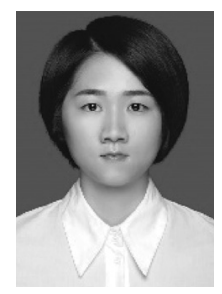

ZHAO Danling was born in 1991. She is a Ph.D. student in National University of Defense Technology. She has published many papers in international magazines, such as Physica A. Her research interests are decision-making analysis, intelligent optimization algorithm, and complex network.

E-mail: zhaodanling11@163.com

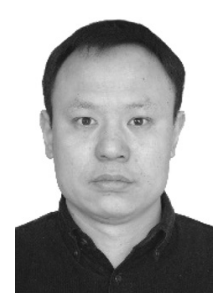

WEI Yong was born in 1987. He received his Ph.D. degree in cartography and geographical information system from Information Engineering University, Zhengzhou, China. He is a data processing and analysis engineer in Unit 31008 of the PLA. His main research interests are data analysis, data mining, and decision making.

E-mail: youwei@163.com 